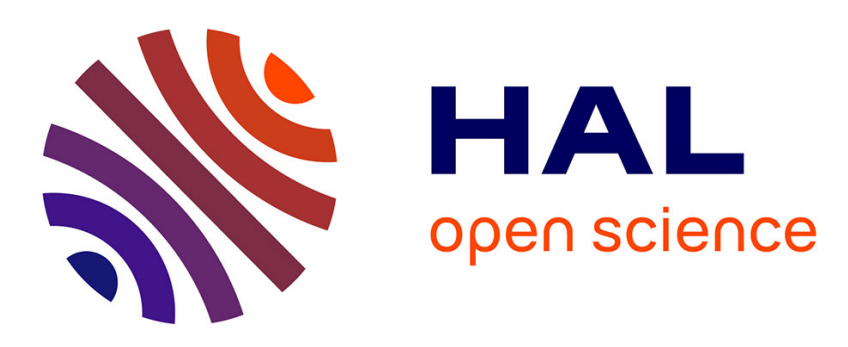

\title{
Solving the Crop Allocation Problem using Hard and Soft Constraints
}

\author{
Mahuna Akplogan, Simon de Givry, Jean-Philippe Metivier, Gauthier \\ Quesnel, Alexandre Joannon, Frédérick Garcia
}

\section{To cite this version:}

Mahuna Akplogan, Simon de Givry, Jean-Philippe Metivier, Gauthier Quesnel, Alexandre Joannon, et al.. Solving the Crop Allocation Problem using Hard and Soft Constraints. RAIRO - Operations Research, 2013, 47 (2), pp.151-172. 10.1051/ro/2013032 . hal-01024641

\section{HAL Id: hal-01024641 https://hal.science/hal-01024641}

Submitted on 16 Jul 2014

HAL is a multi-disciplinary open access archive for the deposit and dissemination of scientific research documents, whether they are published or not. The documents may come from teaching and research institutions in France or abroad, or from public or private research centers.
L'archive ouverte pluridisciplinaire HAL, est destinée au dépôt et à la diffusion de documents scientifiques de niveau recherche, publiés ou non, émanant des établissements d'enseignement et de recherche français ou étrangers, des laboratoires publics ou privés.

$$
\text { Copyright }
$$




\title{
SOLVING THE CROP ALLOCATION PROBLEM USING HARD AND SOFT CONSTRAINTS*
}

\author{
Mahuna Akplogan ${ }^{1}$, Simon de Givry ${ }^{1}$, Jean-Philippe \\ MÉtivier $^{2}$, Gauthier Quesnel ${ }^{1}$, Alexandre Joannon ${ }^{3}$ \\ AND FRÉDÉRICK GARCIA ${ }^{1}$
}

\begin{abstract}
Application tools for the crop allocation problem (CAP) are required for agricultural advisors to design more efficient farming systems. Despite the extensive treatment of this issue by agronomists in the past, few methods tackle the crop allocation problem considering both the spatial and the temporal aspects of the CAP. In this paper, we precisely propose an original formulation addressing the crop allocation planning problem while taking farmers' management choices into account. These choices are naturally represented by hard and soft constraints in the Weighted CSP formalism. We illustrate our proposition by solving a medium-size virtual farm using either a WCSP solver (toulbar2) or an ILP solver (Number Jack/SCIP). This preliminary work foreshadows the development of a decision-aid tool for supporting farmers in their crop allocation strategies.
\end{abstract}

Keywords. Weighted constraint satisfaction problem, integer linear programming, crop allocation problem.

Mathematics Subject Classification. - Please, give AMS classification codes - .

Received January 28, 2013. Accepted February 11, 2013.

* This work is partly funded by the "Agence nationale de la Recherche" ANR-10-BLA-0214.

1 INRA, UR 875 Biométrie et Intelligence Artificielle, 31326 Toulouse.

\{makploga, degivry, gquesnel, fgarcia\}@toulouse.inra.fr

2 GREYC-CNRS, UMR 6072, Université de Caen, 14032 Caen.

jean-philippe.metivier@unicaen.fr

3 INRA, UR 980 SAD-Paysage, 35042 Rennes. joannon@rennes.inra.fr 


\section{INTRODUCTION}

The design of a cropping plan is one of the first steps in the process of crop production and is an important decision that farmers have to take. By cropping plan, we mean the acreage occupied by all the different crops every year and their spatial allocation within a farming land. The cropping plan decision can be summarized as (1) the choice of crops to be grown, (2) determining of all crops' acreage, and (3) their allocation to plots. Despite the apparent simplicity of the decision problem, the cropping plan decisions depend on multiple spatial and temporal factors interacting at different levels of the farm management.

The cropping plan decision-making combines long term planning activities with managerial and operational activities to timely control the crop production process. Modeling a decision-making process supporting such farmers' decisions therefore requires the planning of crop allocation over a finite time horizon, and the need for replanning as the context changes (e.g., weather, prices). In this paper, we precisely focus on the planning task seen as a spatio-temporal crop allocation problem (CAP) whose relevance is assessed by a global objective function. In addition to many approaches based on an optimization procedure, the objective of our work is to propose new directions addressing the crop allocation problem while taking farmers' decision factors into account. These factors are formalized as hard and soft constraints in the WCSP framework. Our choice of physical constraints and farmer's preferences is based on a survey of farmers' processes [11].

Nevertheless, designing cropping plans with such an approach is still an open question due to many other decision factors that could be taken into account to solve the crop allocation problem. This preliminary work foreshadows the implementation of a spatially explicit decision-aid tool, namely CRASH (Crop Rotation and Allocation Simulator using Heuristics), developed for supporting farmers in their crop allocation strategies.

The paper is organized as follows. In Section 2, we describe the crop allocation problem. We introduce some specific definitions and emphasize the problem. Section 3 describes some existing approaches used to design cropping plans, showing their main limitations. In Section 4, we introduce the Weighted CSP formalism. Section 5 describes a WCSP formulation of the crop allocation problem. Its reformulation as an integer linear program is given in Section 6. In Section 7, we illustrate our approach by solving a medium-size virtual farm using either the direct WCSP formulation or a decomposed one, or the ILP reformulation. Finally, we conclude in Section 8.

\section{Crop allocation PRoblem}

\subsection{OVERVIEW OF THE PROBLEM}

We define the crop allocation problem as a spatio-temporal planning problem in which crops are assigned to plots over a finite time horizon $\mathcal{H}$ (Fig. 1). The 


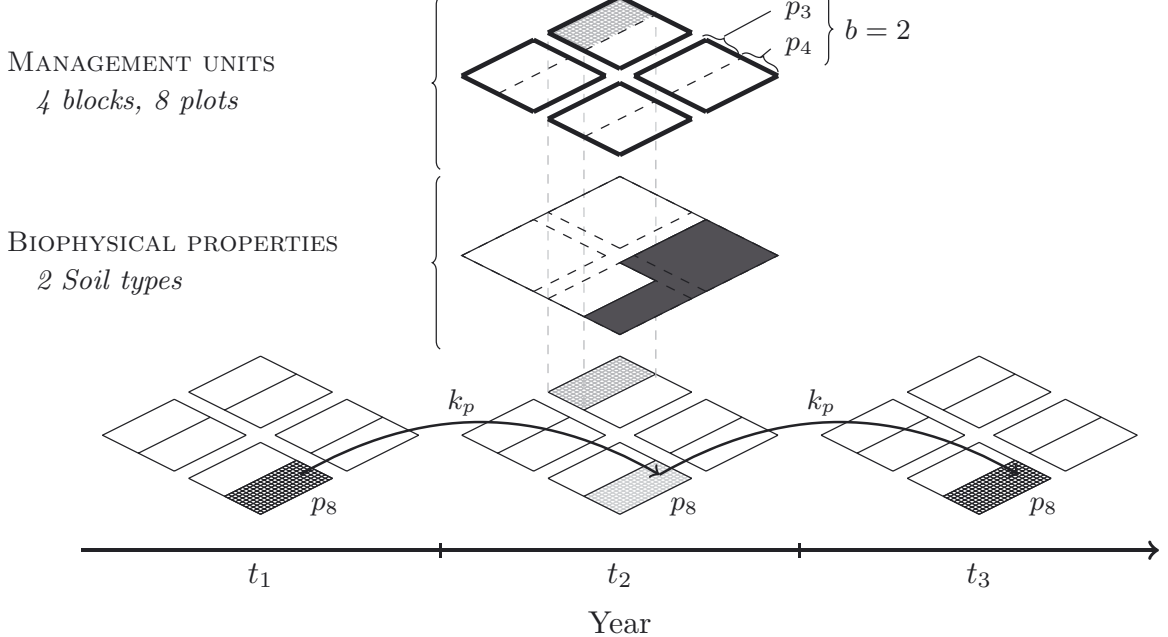

FIGURE 1. Schematic representation of the spatial and temporal aspects of the decision-making problem $\left(t_{i}\right.$ : year, $b$ : block, $p_{j}$ : plot, $k_{p}$ : preceding effect).

planning problem depends on multiple spatial and temporal factors. In space, these factors are organized in many different levels (management units in Fig. 1). These management units are decided by the farmer in order to organize his/her work and allocate resources. In order to simplify our example, we only considered the two main management units: plot $\left(p_{j}\right)$ and block $(b)$. The first concerns the annual management of crops. The spatial configuration of plots are adapted over years in order to enforce the spatial balance of crop acreage. As shown in Figure 1, blocks are subsets of plots managed in a consistent way. Blocks are characterized by one cropping system defined by the same collection of crops and by the use of a consistent set of production techniques applied to these crops (e.g., fertilizer, irrigation water). The delimitation of blocks and plots are not reshaped in the CAP considered in this work. They are mostly defined by the structural properties of the farm such as the availability of resources (e.g., access to irrigation water) and by the biophysical properties (e.g., soil type, accessibility, topography). These biophysical properties are also used to determine if a crop could not be produced in good conditions on certain soil types.

Over time, the repetition of the same crop on the same plot is not allowed or not advisable without facing decrease in soil fertility, or increase in diseases or weeds infestation. We deal with these temporal factors by summarizing the assessment of crop sequence quality in two indicators: the minimum return time of a crop and the preceding effect. The minimum return time of a crop $v$, denoted $r t(v)$, is a hard constraint defined as the minimum number of years before growing the same crop $v$ on the same plot. In Figure 1, the minimum return time of the crop 
produced on $p_{8}$ at $t_{1}$ is equal to two years. More generally let $t, t^{\prime}$ be two different years $\left(t<t^{\prime}\right), p_{j}$ a plot and $v$ a crop, if $p_{j}(t)=p_{j}\left(t^{\prime}\right)=v$ then $\left(t^{\prime}-t\right) \geq r t(v)$.

The preceding effect, denoted $k_{p}$, is a preference indicator representing the effect of the previous crop on the next one [25]. Based on $k_{p}$, some crop sequences can be ignored for their bad effects or recommended for their beneficial effects for production purposes.

Furthermore, some authors [9] have argued that the reproducibility of a cropping system over time is only ensured when crop allocation choices are derived from a finite crop sequence which can be repeated over the time. We therefore introduce the concept of repeatability while looking for such a crop sequence. This means that the proposed crop sequence can be repeated over time without breaking the constraint $r$. We introduce this concept, known as crop rotations, because it is widely used by farmers. It is characterized by a cyclic sequence where the temporal sequence of crops is limited to the order of appearance of crops on the same plot during a fixed period.

\subsection{Description OF THE HARD AND SOFt CONSTRAints}

Solving the crop allocation problem (CAP) is to assign crops (values) to plots (variables) over a finite time horizon $\mathcal{H}$. An assignment of crops must satisfy a given set of constraints. We retained as hard constraints the minimum return time $(r t)$, the history of plots, and the biophysical properties (soil types, resource accessibility). Soft constraints are related to the preceding effects $\left(k_{p}\right)$ and the spatio-temporal balance of crop acreage such that resources are efficiently used. Hard and soft constraints are defined either at the:

- plot level, by expressing the preceding effect and the compatibility of the crop to the biophysical properties, by enforcing the history of crop values and the minimum return time, and by specifying which plots can be split or combined,

- block level, by expressing the spatial and temporal compatibility of crops for all the plots of a block.

- farm level, by expressing farmer preferences or the global use of resources.

Let us consider the crop allocation problem described in Figure 2. In this problem, we consider 4 blocks and 15 plots. The size of the farmland (180 ha) corresponds to a middle size real-world CAP. Four crops are produced over all the blocks: winter wheat $(\mathrm{BH})$, spring barley $(\mathrm{OP})$, maize $(\mathrm{MA})$ and winter rape $(\mathrm{CH})$. Each block has a fixed area (see Fig. 2). Blocks 1 and 3 have access to irrigation equipments $e q_{1}$ and $e q_{2}$. The annual quota of irrigation water over the blocks is $6000 \mathrm{~m}^{3}$ (respectively $4000 \mathrm{~m}^{3}$ ) for $e q_{1}$ (respectively $e q_{2}$ ). Only the maize must be irrigated. There are two different types of soil: type 1 (block 1,3) and type 2 (block 2, 4). The table on Figure 2 shows the sequence of crops produced by each plot during the last five years. 


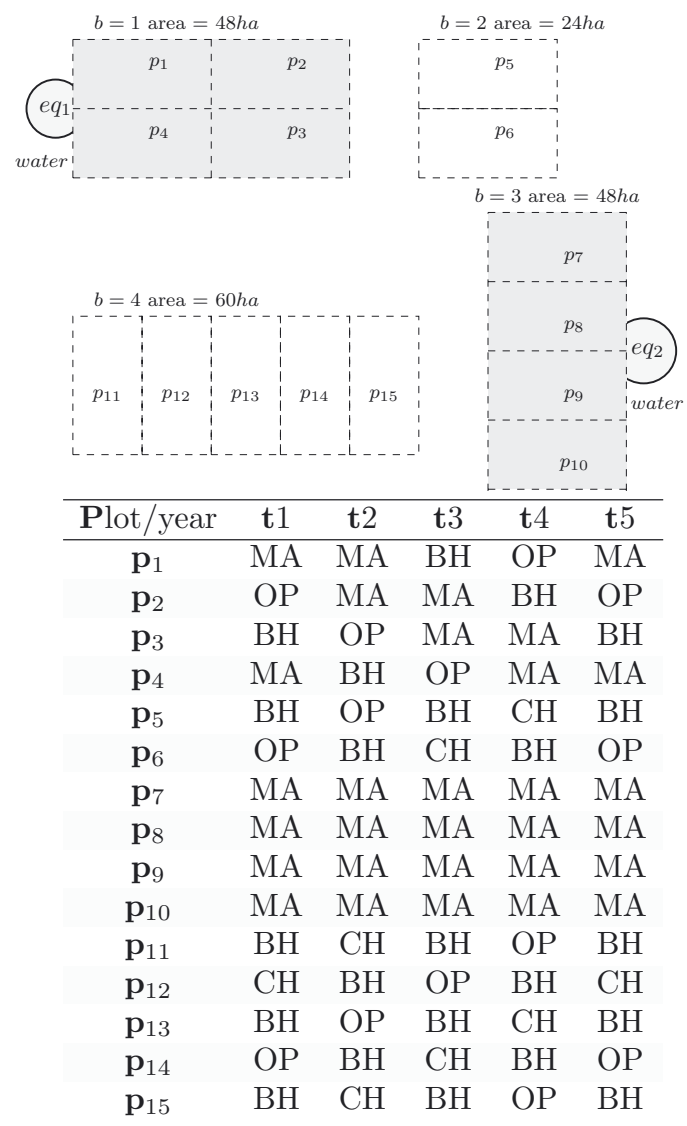

Figure 2. A virtual farm with 4 blocks, 15 plots (12ha per plot). The grey blocks have their own irrigation equipment $\left(e q_{1}, e q_{2}\right)$. The table contains the historic values for each plot over the last five years. These values may violate the minimum return time constraint.

2.2.1. Spatio-Temporal hard constraints

1. h-SCC - Spatial compatibility of crops: some crops are forbidden on some 2 plots. For instance, the crop $\mathrm{CH}$ cannot be assigned to plots whose soil type is 3 1 (blocks 1,3). Its biophysical properties are not favorable to the crop's growth. 4

2. h-EQU - Plot equality: for instance, on block 3 , the plots $p_{7}$ (respectively $p_{9}$ ) 5 and $p_{8}$ (respectively $p_{10}$ ) must be assigned to the same crop every year. Indeed, 6 these plots are decided by the farmer to be managed in the same manner. 7

3. h-HST - Plot history: each plot has some predefined historic values (see the 8 table in Fig. 2). 


\begin{tabular}{c|cccc}
\hline$k_{p}$ & \multicolumn{4}{|c}{ previous crop } \\
& BH & OP & MA & CH \\
\hline BH & 40 & 10 & 10 & 0 \\
OP & 20 & 30 & 10 & 0 \\
MA & 0 & 0 & 30 & 50 \\
CH & 0 & 0 & 50 & 40
\end{tabular}

FigurE 3. Table of preceding effects $\left(k_{p}\right)$.

4. h-SCA - Same crops assigned: over the time, the same collection of crops must be assigned to every plot of the same block.

5. h-TSC - Temporal sequence of crops: for each plot, the minimum return time $r t$ must always be enforced. In our virtual farm example, we use $r t(B H)=2$, $r t(O P)=3, r t(M A)=2$, and $r t(C H)=3$.

6. h-CCS - Cyclicity of crop sequences: for each plot, the crop sequence after the historic values must be endlessly repeatable without violating the minimum return time constraint. This cyclicity constraint on crop sequences, also called crop rotations, is a common farming practice.

7. h-RSC - Resource constraints: a fixed amount of resources is available per year. The resource usage for the plots must not exceed some given capacity constraints. For instance, in Figure 2, we have only one irrigated crop (MA). Knowing that we need $165 \mathrm{~m}^{3}$ of water per hectare, the annual production of MA on block $b=1$ cannot exceed $36.36 h a$.

\subsubsection{Spatio-Temporal soft constraints}

1. s-CSQ - Crop sequence quality: each pair of temporally successive crops is associated to a cost $k_{p}$ that defines its preceding effect. Figure 3 defines all the $k_{p}$ values. A larger cost means a less desirable crop succession.

2. s-TOP - Farm topology: plots where the same crop is assigned must be spatially grouped. By this we mean that it is preferable to group as much as possible the same crop on the same block. Thus, travel time can be reduced as well as the time spent by the farmers on operational activities that control the crop production process. Therefore, every plot having a direct neighbor in the same block assigned to a different crop is penalized by a cost $\delta_{2}$.

3. s-SBC - Spatial balance of crop acreage: a defined acreage of some crops every year. For instance, in the CAP defined above, the acreage of MA should be within the range $[24,48]$ ha on block 1 and $[12,24]$ ha on block 3 . These values are decided by the farmer according to its expected yield. Any deviation is penalized by a cost $\delta_{1}$.

4. S-SGBC - Spatial global balance of crop acreage: it extends the previous preference over all the blocks. For instance, the annual global acreage of MA and $\mathrm{BH}$ over all the blocks should be respectively within the range $[40,72] \mathrm{ha}$ and $[70,100]$ ha. These values are decided by the farmer according to its expected yield. Any deviation is penalized by a cost $\delta_{1}$. 
5. s-TBC - Temporal balance of crop acreage: a defined occurrence of some crops on each plot over the future horizon. In the CAP defined above, between $[1,2]$ occurrences of crop $\mathrm{CH}$ should appear in every crop sequence associated to each plot. These values are decided by the farmer according to the desired crop sequence quality. Any deviation is penalized by a cost $\delta_{3}$.

In practice, we suggest the costs $k_{p}, \delta_{1}, \delta_{2}$, and $\delta_{3}$ take values very distant from each other such that $k_{p}>\delta_{1}>\delta_{2}>\delta_{3}$. By doing so, a simple but realistic hierarchy can be introduced among the soft constraints by means of a weighted sum objective function (defined in Sect. 4). Indeed, first and foremost, the preceding effects $k_{p}$ must be minimized because of their consequences on the next crops. The spatial balance of crop acreage related to $\operatorname{cost} \delta_{1}$, implicitly defines the annual receipts of the farmer. It must be ensured as much as possible. Afterwards the working hours can be reduced by grouping the same crops together $\left(\delta_{2}\right)$. Lastly, the additional preferences related to the temporal balance of crop acreage $\left(\delta_{3}\right)$ can be enforced.

\section{RELATED WORK}

Since Heady [15], the cropping plan decision was represented in most modeling approaches as the search of the best land-crop combination [19]. Objectives for achieving a suitable cropping plan were often based on the complete rationality paradigm using a single monetary criterion optimization, multi-attribute optimization [3] or assessment procedures [5]. In these approaches, the cropping plan decision is mainly represented into models by one of the two concepts, i.e., the cropping acreage $[18,34]$ or crop rotation $[9,12]$. These two concepts are two sides of the cropping plan decision problem, i.e., the spatial and temporal aspects. As described in [11], most of the existing modeling approaches do not combine explicitly the spatial and temporal aspects of the CAP. The cropping plan is not spatially represented and is summarized as simple crop acreage distributions across various land types. At the farm level, the heterogeneity of a farm territory is generally described using soil types as the only criterion. However we can notice that at the farm level (respectively at the landscape level), [35] (resp. [7]) took into account both dimensions, spatial and temporal, but the solutions found were not optimal (using case-based reasoning (resp. simulated annealing algorithm)).

The originality of our approach lies on the consideration of both dimensions, i.e., spatial and temporal while solving the CAP at the farm scale. Many approaches for modeling and solving planning and scheduling problems exist, dealing with spatial and temporal aspects in a quantitative or qualitative manner (e.g., [4]). We choose a quantitative formulation of the CAP into a Weighted CSP (whereas [35] used classical CSPs) and solve it using either a WCSP or an ILP solver.

\section{Weighted CSP fORMalism}

We model the crop allocation problem as a Weighted Constraint Satisfaction 
optimization problems efficiently by giving the list of hard and soft (global) constraints [28]. The WCSP formalism extends the CSP formalism by replacing constraints by cost functions. A WCSP is a triplet $\langle\mathcal{X}, \mathcal{D}, \mathcal{W}\rangle$ where:

- $\mathcal{X}=\{1, \cdots, n\}$ is a set of $n$ variables.

- $\mathcal{D}=\left\{D_{1}, \cdots, D_{n}\right\}$ is a set of finite domains. Each variable $i \in \mathcal{X}$ takes a value in its domain $D_{i} \in \mathcal{D}$.

- $\mathcal{W}=\left\{W_{S_{1}}, \cdots, W_{S_{e}}\right\}$ is a set of $e$ cost functions where $S_{i} \subseteq \mathcal{X}$ be a subset of the variables (i.e., the scope). We denote $l\left(S_{i}\right)$ the set of tuples over $S_{i}$. Each cost function $W_{S_{i}}$ associates an integer cost in $[0, m]$ to every tuple in $l\left(S_{i}\right)$, where $m \in[1,+\infty]$ corresponds to a forbidden assignment. Thus, a hard constraint is modeled by a cost function returning either 0 if the constraint is satisfied or $m$ if it is not. A soft constraint is modeled by a cost function returning either 0 if the constraint is satisfied or a specific cost depending on the violation measure if it is not.

The Weighted Constraint Satisfaction Problem is to find a complete assignment $A \in l(\mathcal{X})$ that minimizes $\sum_{W_{S_{i}} \in \mathcal{W}} W_{S_{i}}\left(A\left[S_{i}\right]\right)$, where $A\left[S_{i}\right]$ is the projection of a tuple on the set of variables $S_{i}$. Exact methods rely on a Depth-First Branch-andBound algorithm, enforcing soft local consistencies in order to prune the search tree $[1,13,21-23,33]$.

\section{Modeling the CROP ALLOCATion PROBlem AS A WCSP}

Let us consider a spatial sampling of the farmland into landunits. A landunit is a piece of indivisible and homogeneous land having the same history and the same biophysical properties. Each landunit has the same size which defines the precision level of the numerical constraints. A plot is a combination of landunits.

Considering our spatial sampling, we define a CAP as a spatio-temporal planning problem in which crops are assigned to landunits over a finite time horizon $\mathcal{H}$. Let $\mathcal{B}$ be a set of blocks and $\mathcal{N}_{b}$ the set of landunits in block $b$. We define the associated WCSP problem as follow.

$\mathcal{X}$ a set of variables $x_{b, i}^{t}$ that define the landunit $i$ in block $b$ at year $t$, with $i \in \mathcal{N}_{b}, b \in \mathcal{B}$, and $t \in[1, \mathcal{H}]$. Thus, each landunit is described by $\mathcal{H}$ variables that represent the landunit occupation over the time horizon. We define $[1, h]$ and $[h+1, \mathcal{H}]$ respectively the past (history) and the future variable times. See Figure 4 for an example with five historic variables (white nodes). The history variables enable us to enforce the return value of crops over the future variables.

$\mathcal{D}$ the domain $D_{b, i}$ of variable $x_{b, i}^{t}$ is the set of possible crops over the landunit $i$ in block $b$. In our virtual farm example, we have $D_{b, i} \subseteq\{B H, O P, M A, C H\}$.

$\mathcal{W}$ the cost functions are divided into five different types of hard and soft constraints: (1) simple table cost functions (arity up to 5), (2) same hard global constraint, (3) regular hard global constraint, (4) gcc hard global cardinality 


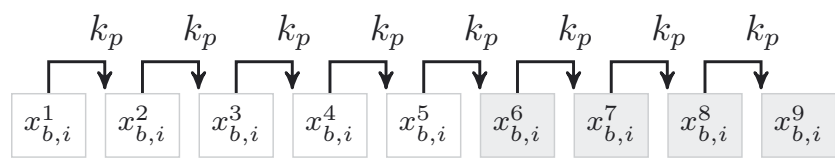

Figure 4. A temporal sequence of 9 variables over landunit $i$ in block $b(\mathcal{H}=9$ and $h=5)$.

constraint, (5) soft-gcc soft global cardinality constraint. These cost functions are precisely defined in the next sections.

1

2

\subsection{Simple COST FunCtions}

3

The hard and soft constraints h-SCC, h-EQU, h-HST, s-CSQ, and s-TOP are defined by:

h-SCC. $\forall t \in[h+1, \mathcal{H}], \forall b \in \mathcal{B}, \forall i \in \mathcal{N}_{b}$, let $W_{x_{b, i}^{t}}^{S C C}$ be a unary cost function associated to the spatial compatibility of crops.

$\forall a \in D_{b, i}$

$$
W_{x_{b, i}^{t}}^{S C C}(a)= \begin{cases}+\infty & \text { if a is forbidden for } \\ & \text { block } b, \text { landunit } i \\ 0 \quad & \text { otherwise }\end{cases}
$$

h-EQU. $\forall t \in[h+1, \mathcal{H}], \forall b \in \mathcal{B}$, for all pairs of landunits $(i, j) \in \mathcal{N}_{b} \times \mathcal{N}_{b}$ that are 10 decided by the farmer to be managed in the same manner, we define an equality 11 constraint $W_{x_{b, i}^{t}, x_{b, j}^{t}}^{E Q U}$ between the two landunits. 12

$\forall a \in D_{b, i}, \forall a^{\prime} \in D_{b, j}$

$$
W_{x_{b, i}^{t}, x_{b, j}^{t}}^{E Q U}\left(a, a^{\prime}\right)= \begin{cases}0 & \text { if } a=a^{\prime} \\ +\infty & \text { otherwise }\end{cases}
$$

h-HST. $\forall t \in[1, h], \forall b \in \mathcal{B}, \forall i \in \mathcal{N}_{b}$, let $W_{x_{b, i}^{t}}^{H S T}$ be unary cost function associated 15 to the historic values of landunits.

$\forall a \in D_{b, i}$

$$
W_{x_{b, i}^{t}}^{H S T}(a)= \begin{cases}0 & \text { if } a=\text { historic }\left(x_{b, i}^{t}\right) \\ +\infty & \text { otherwise }\end{cases}
$$

where historic $\left(x_{b, i}^{t}\right)$ returns the historic value of landunit $i$ in block $b$ at year $t$.

s-CSQ. $\forall t \in[1, \mathcal{H}], \forall b \in \mathcal{B}, \forall i \in \mathcal{N}_{b}$, let $W_{x_{b, i}^{t}, x_{b, i}^{t+1}}^{C S Q}$ be a binary cost function associated to the preceding effect $k_{p}$.

Let $\operatorname{KP}\left(a, a^{\prime}\right)$ be a function that returns the preceding effect $k_{p}$ of doing the crop $a^{\prime}$ after $a$.

$\forall a \in D_{b, i}, \forall a^{\prime} \in D_{b, i}$

$$
W_{x_{b, i}^{t}, x_{b, i}^{t+1}}^{C S Q}\left(a, a^{\prime}\right)=\operatorname{KP}\left(a, a^{\prime}\right)
$$


s-TOP. $\forall t \in[h+1, \mathcal{H}], \forall b \in \mathcal{B}, \forall i \in \mathcal{N}_{b}$, let $W_{S}^{T O P}$ be a 5 -ary cost function associated to the farm land topology. Let neighbor $(i)$ be a neighborhood function which returns the landunits $j \in \mathcal{N}_{b}$ spatially close to $i$. For instance, in our virtual farm example, we consider the 4 nearest neighbors, the so-called von Neumann neighborhood. Here, the scope $S$ is equal to $\left\{x_{b, i}^{t}, x_{b, n}^{t}, x_{b, s}^{t}, x_{b, e}^{t}, x_{b, w}^{t}\right\}$ where landunits $n, s, e, w$ are the 4 nearest neighbors respectively at the North, South, East and West of $i . \forall a \in D_{b, i}, \forall a_{n} \in D_{b, n}, \forall a_{s} \in D_{b, s}, \forall a_{e} \in D_{b, e}, \forall a_{w} \in D_{b, w}$

$$
W_{S}^{T O P}\left(a, a_{n}, a_{s}, a_{e}, a_{w}\right)=\left\{\begin{array}{c}
0 \text { if } a=a_{n}=a_{s}= \\
a_{e}=a_{w} \\
\delta_{2} \text { otherwise }
\end{array}\right.
$$

According to the block structure and the position of the landunit $i$ in its block, the arity of $W_{S}^{T O P}$ may be reduced (corner and border cases).

\subsection{Crop COLlection OVER A BLOCK USING same}

h-SCA. Considering a block $b$, the subset of $(\mathcal{H}-h) *\left|\mathcal{N}_{b}\right|$ future variables $x_{b, i}^{t}$ associated to each landunit $i$ in $b$ must be assigned to the same crop collection. Thus, $\forall(i, j) \in \mathcal{N}_{b} \times \mathcal{N}_{b}$ (with $i \neq j$ ), the set of values assigned to the temporal sequence of variables defining $i$ is a permutation of those of $j$. By using the same constraint introduced in [6] we define h-SCA. For each block $b$, we choose a leading landunit $i$. We then define a $2 \times(\mathcal{H}-h)$-ary cost function $W_{S}^{S C A}$ with the scope $S$ equal to $\left\{x_{b, i}^{h+1}, \cdots, x_{b, i}^{\mathcal{H}}, x_{b, j}^{h+1}, \cdots, x_{b, j}^{\mathcal{H}}\right\}$ for every $j \in \mathcal{N}_{b} \backslash\{i\}$. Let $A\left[x_{b, i}^{h+1}, \cdots, x_{b, i}^{\mathcal{H}}\right]$ and $A\left[x_{b, j}^{h+1}, \cdots, x_{b, j}^{\mathcal{H}}\right]$ denote the two sub-assignments of the variables in $S$. The constraint $W_{S}^{S C A}$ requires that $A\left[x_{b, i}^{h+1}, \cdots, x_{b, i}^{\mathcal{H}}\right]$ is a permutation of $A\left[x_{b, j}^{h+1}, \cdots, x_{b, j}^{\mathcal{H}}\right]$. For instance, $(\mathrm{CH}-\mathrm{OP}-\mathrm{CH}-\mathrm{BH}, \mathrm{CH}-\mathrm{BH}-\mathrm{OP}-\mathrm{CH})$ is a valid pair of crop sequences for same. Note that for a given block, h-EQU implies h-SCA.

$$
W_{S}^{S C A}=\operatorname{same}(\underbrace{x_{b, i}^{h+1}, \cdots, x_{b, i}^{\mathcal{H}}}_{i}, \underbrace{x_{b, j}^{h+1}, \cdots, x_{b, j}^{\mathcal{H}}}_{j})
$$

\subsection{CRop SEQUence USING regular}

The constraints h-TSC and h-CCS are related to temporal crop sequences. We represent them by using the regular constraint [30]. $\forall b \in \mathcal{B}, \forall i \in \mathcal{N}_{b}, \forall a \in D_{b, i}$, let $M_{b, i}^{a}$ be a finite automaton, $\mathcal{L}\left(M_{b, i}^{a}\right)$ the language defined by $M_{b, i}^{a}$, and $S_{b, i}$ a temporal sequence of $\mathcal{H}$ variables that describes landunit $i$ of block $b$ over the horizon. An assignment $A\left[S_{b, i}\right]$ satisfies a regular $\left(S_{b, i}, M_{b, i}^{a}\right)$ constraint iff $A\left[S_{b, i}\right] \in$ $\mathcal{L}\left(M_{b, i}^{a}\right)$. 


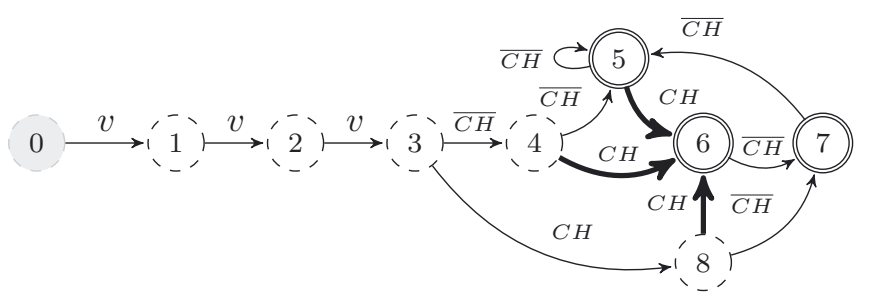

Figure 5. Automaton for crop $a=C H$ with $r t(C H)=3$ and $h=5 . v$ denotes any value in $D_{b, i}$. The notation $\overline{C H}$ corresponds to $D_{b, i} \backslash\{C H\}$. The associated language accepts every pattern over the historic variables and only the patterns that enforce the minimum return time in the future variables taking into account the historic values. e.g., $\mathrm{CH}-\mathrm{OP}-\mathrm{CH}-\mathrm{OP}-\mathrm{CH}-\mathrm{BH}-\mathrm{OP}-\mathrm{CH}-\mathrm{BH}$ is a valid crop sequence for $\mathcal{H}=9$ and $h=5$.

h-TSC. Considering each landunit $x_{b, i}$, the crop sequence is enforced by defining for each crop $a \in D_{b, i}$ a language $\mathcal{L}\left(M_{b, i}^{a}\right)$ such that the same value $a$ is assigned to $x_{b, i}^{t}$ and $x_{b, i}^{t^{\prime}}\left(t^{\prime}>t\right)$ only if $x_{b, i}^{t^{\prime}}$ enforces the minimum return time $r t(a)$ i.e., $t^{\prime} \geq t+r t(a)$. We define regular $\left(S_{b, i}, M_{b, i}^{a}\right)$ where $M_{b, i}^{a}$ is described as in Figure 5 for crop $a=C H$ the minimum return time of which is $\operatorname{rt}(\mathrm{CH})=3$ years. Here, the initial state is 0 while final states are $5,6,7$. Arcs are labelled with crop values.

As shown by the automaton in Figure 5, the historic variables are used to enforce the minimum return time over the future variables. We then define an $\mathcal{H}$-ary cost function $W_{S_{b, i}}^{T S C^{a}}$ associated to each pair of landunit $i$ in block $b$ and each crop $a$ such that:

$$
\forall b \in \mathcal{B}, \forall i \in \mathcal{N}_{b}, \forall a \in D_{b, i}
$$

$$
W_{S_{b, i}}^{T S C^{a}}=\operatorname{regular}\left(x_{b, i}^{1}, \cdots, x_{b, i}^{\mathcal{H}}, M_{b, i}^{a}\right)
$$

h-CCS. Considering each landunit $x_{b, i}$, we combine h-TSC with a repeatability constraint also defined by a set of regular constraints. The constraint h-CCS ensures that any crop sequence assignment after the historic values can be endlessly repeated without violating the minimum return time constraint h-TSC. Figure 6 describes a cyclic automaton for crop $C H$. The initial state is 0 while final states are $3,6,9,12$. The scope of the cost function $W_{S_{b, i}}^{C C S^{a}}$ is restricted to future variables:

$$
\forall b \in \mathcal{B}, \forall i \in \mathcal{N}_{b}, \forall a \in D_{b, i}
$$

$$
W_{S_{b, i}}^{C C S^{a}}=\operatorname{regular}\left(x_{b, i}^{h+1}, \cdots, x_{b, i}^{\mathcal{H}}, M_{b, i}^{a}\right)
$$

\subsection{Resource CAPACITy CONSTRAints Using gcc}

In CAP, each landunit consumes a fixed amount of resources according to some qualitative (crop type) and numerical (the area of landunits, the irrigation dose) 


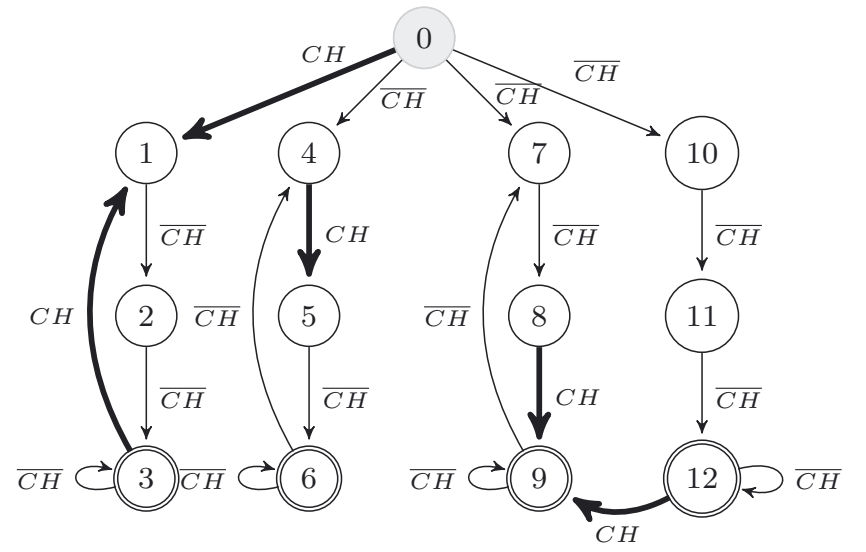

Figure 6. Cyclic automaton for crop $a=\mathrm{CH}$ with $\operatorname{rt}(\mathrm{CH})=3$ and $\mathcal{H}-h \geq 3$. e.g., OP-CH-OP is a valid cyclic sequence for $\mathcal{H}-h=3$ future variables that can be repeated without violating the minimum return time in order to construct a valid sequence for a larger horizon.

requirements. For instance, the maize (MA) is an irrigated crop whereas winter wheat $(\mathrm{BH})$ does not need irrigation. A classical approach dealing with resources is to solve a shortest path problem with resource constraints [17]. The problem is NP-hard if the path needed is elementary. Loosely, solving a resource allocation problem involves both sequencing and counting reasoning. We assume this problem can be reduced to a counting problem under hypotheses 1 and 2 .

Hypothesis 1. Resources are supposed to be usable and systematically renewed every year without doing anything (e.g., annual quota of irrigation water).

This hypothesis is closed to a real CAP because farmers usually have a fixed quota of irrigation water. This is also the case for the working hours capacity constraint in a year if work regulations was taken into account.

Hypothesis 2. $\forall t \in[1, \mathcal{H}], \forall\left(b, b^{\prime}\right) \in \mathcal{B} \times \mathcal{B}$ a pair of blocks, $\forall(i, j) \in \mathcal{N}_{b} \times \mathcal{N}_{b}^{\prime}$ a pair of landunits. The areas of landunits $i$ of block $b$ and $j$ of block $b^{\prime}$ can be considered equivalent according to the problem size.

We make the assumption that the spatial sampling of the farm land into landunits is homogeneous. Under these hypotheses the annual resource allocation is seen as a counting problem at every time $t \in[h+1, \mathcal{H}]$. Thus, given annual resource capacities, we define for each time $t \in[h+1, \mathcal{H}]$ a lower and an upper bound to the number of variables $x_{i, b}^{t}$ that are assigned to a given crop according to both structural and numerical requirements. 
h-RSC. In order to enforce resource capacity constraints h-RSC, we use the global 1 cardinality constraint gcc [32] over the assignments of crops to landunits.

$\forall t \in[h+1, \mathcal{H}]$, let $W_{S_{b}^{t}}^{R S C}$ be a $\left|\mathcal{N}_{b}\right|$-ary global constraint associated to resource capacities.

Given $S_{b}^{t}=\left(x_{b, 1}^{t}, \cdots, x_{b,\left|\mathcal{N}_{b}\right|}^{t}\right)$ the global cardinality constraint (gcc) specifies, for each value $a \in \bigcup D_{b, i}$, a lower bound $l b(a)$ and an upper bound $u b(a)$ to the number of variables in $S_{b}^{t}$ that are assigned to value $a$.

$$
W_{S_{b}^{t}}^{R S C}=\operatorname{gcc}\left(S_{b}^{t}, l b, u b\right)
$$

has a solution if there exists an assignment of $S_{b}^{t}$ such that

$$
\forall a \in \bigcup D_{b, i}, l b(a) \leq\left|\left\{x_{b, i}^{t} \in S_{b}^{t} \mid x_{b, i}^{t}=a\right\}\right| \leq u b(a)
$$

For instance, let us consider the block $b=1$ of the virtual farm described in 11 Figure 2. Assuming that each plot is divided into 8 landunits and $165 \mathrm{~m}^{3}$ of water 12 are needed to produce one hectare of maize (MA), the minimum and maximum 13 annual area dedicated to this crop are respectively 0 and $36.36 \mathrm{ha}$. Thus, over 14 the block $b=1$, the lower bound of variables $x_{b, i}^{t}$ that are assigned to maize is 15 $l b(M A)=8 \times\left\lceil\frac{0 h a}{12 h a}\right\rceil=0$. The upper bound is $u b(M A)=8 \times\left\lfloor\frac{36.36 h a}{12 h a}\right\rfloor=24 . \quad 16$

\subsection{Spatio-Temporal Balance of Crops using Soft-GCC}

Preferences related to the spatio-temporal balance of crops (s-SBC, s-SGBC, and s-TBC) are defined as soft global cardinality constraints (soft-gcc) that allow the violation of both lower and upper bounds of the associated hard constraint gcc.

Given a soft-gcc $(S, l b, u b, \delta)$. Let us define for all $a \in \bigcup D_{b, i}$.

$$
\begin{aligned}
& \operatorname{ovf}(S, a)=\max \left(\left|\left\{x_{b, i}^{t} \mid x_{b, i}^{t}=a\right\}\right|-u b(a), 0\right) \\
& u n f(S, a)=\max \left(l b(a)-\left|\left\{x_{b, i}^{t} \mid x_{b, i}^{t}=a\right\}\right|, 0\right)
\end{aligned}
$$

where $l b$ and $u b$ are respectively the lower and upper bounds for each crop, and $\delta$ a cost. We use the variable-based violation measure $\mu$ (see [16]) which is the minimum number of variables whose values must be changed in order to satisfy the associated gcc constraint. If $\sum_{a \in \bigcup D_{b, i}} l b(a) \leq|S| \leq \sum_{a \in \cup D_{b, i}} u b(a)$, the variable based violation $\mu$ can be expressed by:

$$
\mu(S)=\max \left(\sum_{a \in \bigcup D_{b, i}} \operatorname{ovf}(S, a), \sum_{a \in \bigcup D_{b, i}} \operatorname{unf}(S, a)\right)
$$

The cost function associated to each soft- $\operatorname{gcc}(S, l b, u b, \delta)$ constraint is then $W=$ $\mu(S) \times \delta$. Based on this definition the constraints s-SBC, s-SGBC, and s-TBC are formalized as follow. 
s-SBC. $\forall t \in[h+1, \mathcal{H}], \forall b \in \mathcal{B}$. Let $W_{S_{b}^{t}}^{S B C}$ be a $\left|\mathcal{N}_{b}\right|$-ary cost function soft-gcc associated to block $b$ at time $t$. The scope $S_{b}^{t}=\left\{x_{b, i}^{t} \mid i \in \mathcal{N}_{b}\right\}$.

$$
W_{S_{b}^{t}}^{S B C}=\operatorname{soft}-\operatorname{gcc}\left(S_{b}^{t}, l b, u b, \delta=\delta_{1}\right)
$$

s-SGBC. $\forall t \in[h+1, \mathcal{H}]$. Let $W_{S^{t}}^{S G B C}$ be a $\sum_{b \in \mathcal{B}}\left|\mathcal{N}_{b}\right|$-ary cost function soft-gcc associated to all the landunits at time $t$. The scope $S^{t}=\left\{x_{b, i}^{t} \mid i \in \mathcal{N}_{b}, b \in \mathcal{B}\right\}$.

$$
W_{S^{t}}^{S G B C}=\operatorname{soft}-\operatorname{gcc}\left(S^{t}, l b, u b, \delta=\delta_{1}\right)
$$

s-TBC. $\forall b \in \mathcal{B}, \forall i \in \mathcal{N}_{b}$. Let $W_{S_{b, i}}^{T B C}$ be a $(\mathcal{H}-h)$-ary cost function soft-gcc associated to each landunit $i$. The scope $S_{b, i}=\left\{x_{b, i}^{h+1}, \cdots, x_{b, i}^{\mathcal{H}}\right\}$.

$$
W_{S_{b, i}}^{T B C}=\operatorname{soft}-\operatorname{gcc}\left(S_{b, i}, l b, u b, \delta=\delta_{3}\right)
$$

For instance, considering the temporal balance of crop acreages described in Section 2.2.2, between $[1,2]$ occurrences of winter rape $(\mathrm{CH})$ should appear in every crop sequence associated to each plot. For each sequence of variables $S_{b, i}$ associated to a given landunit, the minimum number of variables in $S_{b, i}$ that should be assigned to winter rape is $l b(C H)=\left\lceil\frac{12 h a}{12 h a}\right\rceil=1$. The maximum is $u b(C H)=\left\lfloor\frac{24 h a}{12 h a}\right\rfloor=2$.

\section{INTEGER LINEAR PROGRAMMING REFORMULATION}

The resulting WCSP can also be represented as an integer linear programming problem using the encoding proposed in [20] and recently tested in [2]. For every landunit variable $x_{b, i}^{t}$ and crop value $a$, there is a boolean variable $x_{b, i}^{t, a}$ which is equal to 1 iff $x_{b, i}^{t}=a$ in the WCSP. Additional constraints enforce that exactly one crop value is selected for each landunit variable. Additional positive integer variables are used to encode nonlinear hard and soft constraints. For instance, in the case of s-CSQ, for every pair of values $\left(a, a^{\prime}\right)$ of successive variables $\left(x_{b, i}^{t}, x_{b, i}^{t+1}\right)$ involved in a binary cost function, there is a boolean variable $c s q_{b, i}^{t, a, a^{\prime}}$ which is equal to 1 iff the pair $\left(x_{b, i}^{t}=a, x_{b, i}^{t+1}=a^{\prime}\right)$ is used. Constraints enforce that a pair is used iff the corresponding values are used. Then, the CAP reduces to the following ILP:

$$
\min \left(\begin{array}{c}
\left(\sum_{t, b, i, a, a^{\prime}} \mathrm{KP}\left(a, a^{\prime}\right) \times c s q_{b, i}^{t, a, a^{\prime}}\right)+\left(\sum_{t, b, i, a} \delta_{2} \times t o p_{b, i}^{t, a}\right) \\
+\left(\sum_{t, b} \delta_{1} \times s b c_{b}^{t}\right)+\left(\sum_{t} \delta_{1} \times s g b c^{t}\right)+\left(\sum_{b, i} \delta_{3} \times t b c_{b, i}\right)
\end{array}\right)
$$


s.t.

\begin{tabular}{|c|c|c|c|}
\hline$\sum_{a \in D_{b, i}} x_{b, i}^{t, a}$ & $=1$ & $\begin{array}{l}\text { domain constraint } \\
\left(\forall t \in[1, \mathcal{H}], b \in \mathcal{B}, i \in \mathcal{N}_{b}\right)\end{array}$ & \\
\hline$x_{b, i}^{t, a}$ & $=0$ & $\left(\forall t \in[h+1, \mathcal{H}], b \in \mathcal{B}, i \in \mathcal{N}_{b}, a \notin D_{b, i}\right)$ & $h-S C C$ \\
\hline$x_{b, i}^{t, a}$ & $=x_{b, j}^{t, a}$ & $\left(\forall t \in[h+1, \mathcal{H}], b \in \mathcal{B}, i, j \in \mathcal{N}^{\prime}{ }_{b}, a \in D_{b, i}\right)$ & $h-E Q U$ \\
\hline$x_{b, i}^{t, \text { historic }\left(x_{b, i}^{i}\right)}$ & $=1$ & $\left(\forall t \in[1, h], b \in \mathcal{B}, i \in \mathcal{N}_{b}\right)$ & $h-H S T$ \\
\hline$\sum_{a^{\prime} \in D_{b, i}} \operatorname{csq} q_{b, i}^{t, a, a^{\prime}}$ & $=x_{b, i}^{t, a}$ & $\left(\forall t \in[1, \mathcal{H}-1], b \in \mathcal{B}, i \in \mathcal{N}_{b}, a \in D_{b, i}\right)$ & $s-C S Q$ \\
\hline$\sum_{a \in D_{b, i}} c s q_{b, i}^{t, a, a^{\prime}}$ & $=x_{b, i}^{t+1, a}$ & $\left(\forall t \in[1, \mathcal{H}-1], b \in \mathcal{B}, i \in \mathcal{N}_{b}, a^{\prime} \in D_{b, i}\right)$ & $s-C s$ \\
\hline
\end{tabular}

$n_{i} \times \operatorname{top}_{b, i}^{t, a}+\sum_{j \in\{i\} \cup \text { neighbor }(i)} x_{b, j}^{t, a} \geq n_{i}\left(\forall t \in[h+1, \mathcal{H}], b \in \mathcal{B}, i \in \mathcal{N}_{b}, a \in D_{b, i}\right)$ $s-T O P$

$\sum_{t \in[h+1, \mathcal{H}]} x_{b, i}^{t, a}=\sum_{t \in[h+1, \mathcal{H}]} x_{b, j}^{t, a} \quad\left(\forall b \in \mathcal{B}, i, j \in \mathcal{N}_{b}, a \in D\right)$ $h-S C A$

$x_{b, i}^{t, a}+x_{b, i}^{t^{\prime}, a}$

$\leq 1 \quad\left(\forall b \in \mathcal{B}, i \in \mathcal{N}_{b}, a \in D_{b, i}\right.$,

$t \in[\max (1, h+2-r t(a)), H-1]$,

$t^{\prime} \in[\max (h+1, t+1)$,

$\min (H, t+r t(a)-1)]) h-T S C$

$x_{b, i}^{t, a}+x_{b, i}^{t^{\prime}, a}$

$\leq 1 \quad\left(\forall b \in \mathcal{B}, i \in \mathcal{N}_{b}, a \in D_{b, i}\right.$,

$t \in[\max (h+1, H+1-r t(a)), H]$,

$t^{\prime} \in[h+1$,

$\min (H, h+r t(a)-1-H+t)]) h-C C S$

$l b_{R S C}(a) \leq \sum_{i \in \mathcal{N}_{b}} x_{b, i}^{t, a} \leq u b_{R S C}(a)\left(\forall t \in[h+1, \mathcal{H}], b \in \mathcal{B}^{\prime}, a \in D^{\prime}\right) h-R S C$

$\operatorname{sbcovf} f_{b}^{t, a} \geq \sum_{i \in \mathcal{N}_{b}} x_{b, i}^{t, a}-u b_{S B C}(a)\left(\forall t \in[h+1, \mathcal{H}], b \in \mathcal{B}^{\prime}, a \in D^{\prime}\right) s-S B C$ $\operatorname{sbcunf}_{b}^{t, a} \geq l b_{S B C}(a)-\sum_{i \in \mathcal{N}_{b}} x_{b, i}^{t, a}\left(\forall t \in[h+1, \mathcal{H}], b \in \mathcal{B}^{\prime}, a \in D^{\prime}\right) s-S B C$ $s b c_{b}^{t} \quad \geq \sum_{a \in D^{\prime}} \operatorname{sbcovf} f_{b}^{t, a} \quad\left(\forall t \in[h+1, \mathcal{H}], b \in \mathcal{B}^{\prime}\right) \quad s-S B C$ $s b c_{b}^{t} \quad \geq \sum_{a \in D^{\prime}} \operatorname{sbcun} f_{b}^{t, a} \quad\left(\forall t \in[h+1, \mathcal{H}], b \in \mathcal{B}^{\prime}\right) \quad s-S B C$

sgbcovf $f^{t, a} \geq \sum_{b \in \mathcal{B}, i \in \mathcal{N}_{b}} x_{b, i}^{t, a}-u b_{S G B C}(a)\left(\forall t \in[h+1, \mathcal{H}], a \in D^{\prime}\right) s-S G B C$ $\operatorname{sgbcunf}^{t, a} \geq \operatorname{lb}_{S G B C}(a)-\sum_{b \in \mathcal{B}, i \in \mathcal{N}_{b}} x_{b, i}^{t, a} \quad\left(\forall t \in[h+1, \mathcal{H}], a \in D^{\prime}\right) s-S G B C$ $\operatorname{sgbc}^{t} \geq \sum_{a \in D^{\prime}} \operatorname{sgbcovf} f^{t, a} \quad(\forall t \in[h+1, \mathcal{H}]) \quad s-S G B C$ $\operatorname{sgbc}^{t} \geq \sum_{a \in D^{\prime}} \operatorname{sgbcunf}^{t, a} \quad(\forall t \in[h+1, \mathcal{H}]) \quad s-S G B C$

$\operatorname{tbcovf} f_{b, i}^{a} \geq \sum_{t \in[h+1, \mathcal{H}]} x_{b, i}^{t, a}-u b_{T B C}(a)\left(\forall b \in \mathcal{B}^{\prime}, i \in \mathcal{N}_{b}, a \in D^{\prime}\right) s-T B C$ $\operatorname{tbcunf} f_{b, i}^{a} \geq l b_{T B C}(a)-\sum_{t \in[h+1, \mathcal{H}]} x_{b, i}^{t, a} \quad\left(\forall b \in \mathcal{B}^{\prime}, i \in \mathcal{N}_{b}, a \in D^{\prime}\right) s-T B C$ $\begin{array}{llll}t b c_{b, i} & \geq \sum_{a \in D^{\prime}} \operatorname{tbcovf} f_{b, i}^{a} & \left(\forall b \in \mathcal{B}^{\prime}, i \in \mathcal{N}_{b}\right) & s-T B C \\ t b c_{b, i} \geq \sum_{a \in D^{\prime}} \text { tbcunf } f_{b, i}^{a} & \left(\forall b \in \mathcal{B}^{\prime}, i \in \mathcal{N}_{b}\right) & s-T B C\end{array}$

with $D=\bigcup_{b, i} D_{b, i}, D^{\prime} \subseteq D, \mathcal{B}^{\prime} \subseteq \mathcal{B}, \mathcal{N}^{\prime}{ }_{b} \subseteq \mathcal{N}_{b}$, and $n_{i}=|\operatorname{neighbor}(i)|+1 . \quad$ 8 The continuous relaxation of this integer linear programming model is known do 9 be the dual of the LP problem encoded by Optimal Soft Arc Consistency [8]. When 
the upper bound $m$ is infinite, OSAC is known to be stronger than any other soft arc consistency and especially stronger than the default Existential Directional Arc Consistency (EDAC) [13] used in the WCSP solver toulbar2. However, as soon as the upper bound $m$ decreases to a finite value, soft local consistencies may prune values and EDAC becomes incomparable with OSAC [2].

\section{EXPERIMENTAL RESUltS}

\subsection{CAP instances}

We performed experiments on four instances of the virtual farm presented in Figure 2. All the hard and soft constraints have been derived from $[10,11]$ who analyzed thirty irrigated arable farms from three different regions in France to study their decision-making about crop allocation. Each instance corresponds to a different sampling of landunits. The total number of landunits is increased from 15 to $120(15,30,60,120)$. For the CAP instance denoted B1234-LU15* with 15 landunits, $\left|\mathcal{N}_{1}\right|=\left|\mathcal{N}_{3}\right|=4,\left|\mathcal{N}_{2}\right|=2$ and $\left|\mathcal{N}_{4}\right|=5$ where $\left|\mathcal{N}_{i}\right|$ denotes the number of landunits in block $i$. In this problem, sampling is done such that the plots are equal to the landunits (12 ha per landunit, see Fig. 2). These landunits are gradually refined by splitting them into 2,4 and 8 smaller ones, to respectively build the instances B1234-LU30*, B1234-LU60*, and B1234-LU120* with 30, 60, and 120 landunits respectively. These samplings are chosen to be representative of different farm sizes. The planning horizon $\mathcal{H}=9$. The four last years are dedicated to the future while the five first are historic ones $(h=5)$. We use the historic values presented in Figure 2.

The costs associated to s-TOP, s-SBC (the same cost is used for s-SGBC), and s-TBC are respectively $\delta_{2}=2, \delta_{1}=100$ and $\delta_{3}=10$. We used the costs of preceding effects $k_{p}$ as given in Figure 3 .

All the CAP instances (in direct, decomposable, or ILP formulation) are available in the cost function benchmark repository ${ }^{4}$.

Computations were performed on a single core of an Intel T9600 at $2.8 \mathrm{GHz}$, using a laptop with 4 GB of RAM, running Linux 2.6, and a 10-hour time-out.

\subsection{Comparison Between the Direct And the Decomposed Weighted CSP FORMULATIONS}

We compared the direct WCSP formulation given in Section 5 with a formulation where all the global cost functions have been decomposed into ternary (or less) cost functions by adding intermediate variables as described in [1]. For the REGULAR constraint, the decomposition is done in such a way that enforcing Directional Arc Consistency [8] on the decomposition achieves the same level of local

\footnotetext{
${ }^{4}$ http://mulcyber.toulouse.inra.fr/scm/viewvc.php/trunk/crafted/CAP/? root=costfunctionlib
} 
TABLE 1. Search effort for proving optimality.

\begin{tabular}{lccccccc}
\hline & $n$ & $e$ & Optimum & \multicolumn{2}{c}{ Direct } & \multicolumn{2}{c}{ Decomposed } \\
& & & & Time(s) & Nodes & Time(s) & Nodes \\
\hline B1234-LU15* & 135 & 465 & 1,824 & 15.64 & 236 & $\mathbf{0 . 1 1}$ & 24 \\
B1234-LU30* $^{2}$ & 270 & 922 & 3,660 & 103.96 & 759 & $\mathbf{0 . 4 0}$ & 65 \\
B1234-LU60* & 540 & 1,804 & 7,492 & 30,624 & 129,592 & $\mathbf{2 . 1 4}$ & 190 \\
B1234-LU120* & 1,080 & 3,568 & 14,800 & - & - & $\mathbf{2 6 . 4 5}$ & 3,386 \\
\hline
\end{tabular}

consistency on the original global cost function [1]. But for SAME, GCC, and SOFTGCC, this result is no more applicable because the decomposition is not Bergeacyclic (our decomposition of these non Berge-acyclic global cost functions uses one AMONG decomposable cost function per crop value).

We solved the CAP instances using the WCSP solver toulbar2 version 0.9. $6^{5}$ with variable elimination of functional (intermediate) variables in preprocessing (option $-f=1$ ), and other default options, including EDAC for binary [13], ternary [33], and global cost functions, using a flow-based algorithm [21-23] for the direct formulation or a built-in decomposition [1] for the decomposed formulation.

Table 1 reports for each instance the number of variables $n$ and cost functions $e$ in the direct WCSP formulation, its optimum, the CPU-time in seconds and number of search nodes for proving optimality for the direct and the decomposed formulations. The initial upper bound was set to the optimum value plus one in order to check the results and be less dependent on the value ordering search heuristics.

The decomposed approach was more than two orders of magnitude faster than the direct approach. This can be explained by the excellent incrementality of usual consistency enforcing algorithms applied to small-arity cost functions compared to the monolithic flow-based algorithms used by the direct formulation.

\subsection{Comparison between the decomposed WCSP And the integer 21 LINEAR PROGRAMMING FORMULATIONS

We compared the decomposed WCSP formulation we have just described with 23 the integer linear programming formulation given in Section 6. We used the ILP 24 solver SCIP version 1.2.0 $0^{6}$ with default options, modeling the ILP formulation us- 25 ing the Python multi-solver interface offered by Number Jack version 0.1.10-11-24 . 26 toulbar2 used variable elimination of functional variables and no initial upper 27 bound. We measured the search effort for finding the optimum and proving opti- 28 mality as reported in Table 2 .

\footnotetext{
${ }^{5}$ http://mulcyber.toulouse.inra.fr/projects/toulbar2

6 http://scip.zib.de

${ }^{7}$ http://numberjack.ucc.ie/
} 
TABLE 2. Search effort for finding the optimum and proving optimality.

\begin{tabular}{lccccccc}
\hline & $n$ & $e$ & Optimum & \multicolumn{2}{c}{ Decomposed } & \multicolumn{2}{c}{ ILP } \\
& & & & Time(s) & Nodes & Time(s) & Nodes \\
\hline B1234-LU15* & 135 & 465 & 1,824 & $\mathbf{0 . 1 1}$ & 38 & 0.12 & 1 \\
B1234-LU30* & 270 & 922 & 3,660 & $\mathbf{0 . 4 0}$ & 92 & 0.79 & 2 \\
B1234-LU60* & 540 & 1,804 & 7,492 & $\mathbf{2 . 1 9}$ & 210 & 2.64 & 8 \\
B1234-LU120* & 1,080 & 3,568 & 14,800 & 23.90 & 3,153 & $\mathbf{7 . 2 5}$ & 9 \\
\hline
\end{tabular}
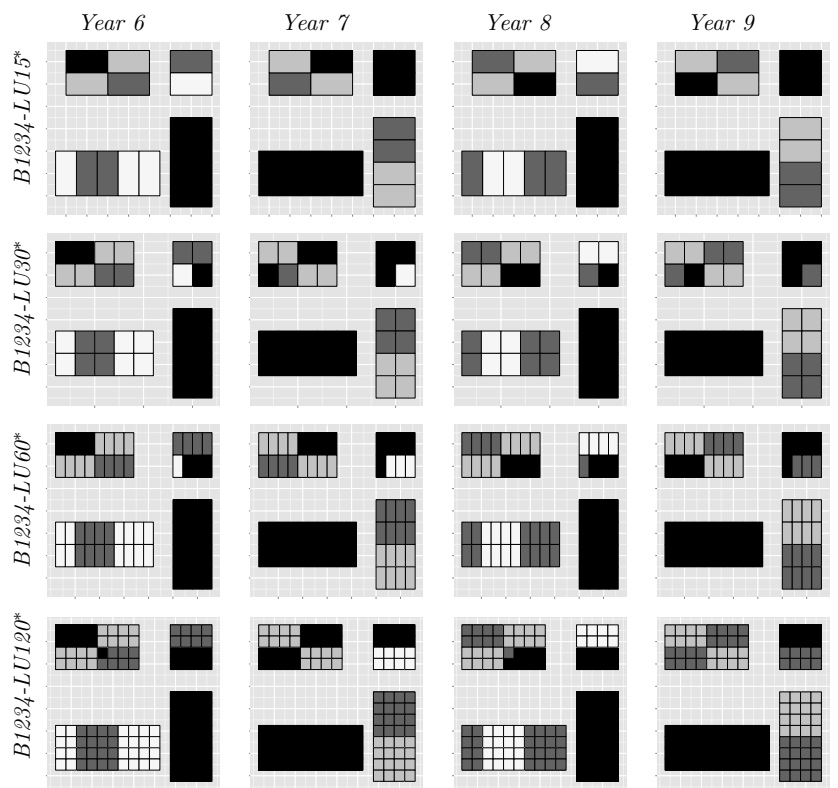

Figure 7. Crop allocation : Winter wheat, Spring barley, Maize, Winter rape.

On the largest instance, SCIP was more than three times faster than toulbar2 and used $160 \mathrm{MB}$ of RAM compared to $803 \mathrm{MB}$ for toulbar2, showing that our decomposed formulation does not scale well compared to the ILP approach.

Let us consider the optimal solutions found by the decomposed approach. Figure 7 represents for each instance the spatio-temporal crop allocation. The minimum return times of crops are enforced. The temporal balance of winter rape is enforced on block 2 and 4 . Due to the historic values in block 3 , the spatial balance of maize is not enforced when the year $t \in\{6,8\}$.

\subsection{Finding ALL THE OPtimal SOLUTiONS}

We also measured the search effort done by toulbar2 on the decomposed formulation to find all the optimal solutions by setting the initial upper bound to the 
TABLE 3. Search effort for finding all the optimal solutions.

\begin{tabular}{lcccccc}
\hline & $n$ & $e$ & Optimum & $\begin{array}{c}\text { Number of } \\
\text { optimal sol. }\end{array}$ & \multicolumn{2}{c}{ Decomposed } \\
& & & & 2 & 0.12 & 58 \\
Time(s) & Nodes \\
\hline B1234-LU15* & 135 & 465 & 1,824 & 12 & 0.39 & 96 \\
B1234-LU30* & 270 & 922 & 3,660 & 136 & 2.45 & 678 \\
B1234-LU60* & 540 & 1,804 & 7,492 & 18 & 30.6 & 7,404 \\
B1234-LU120* & 1,080 & 3,568 & 14,800 & 18 \\
\hline
\end{tabular}

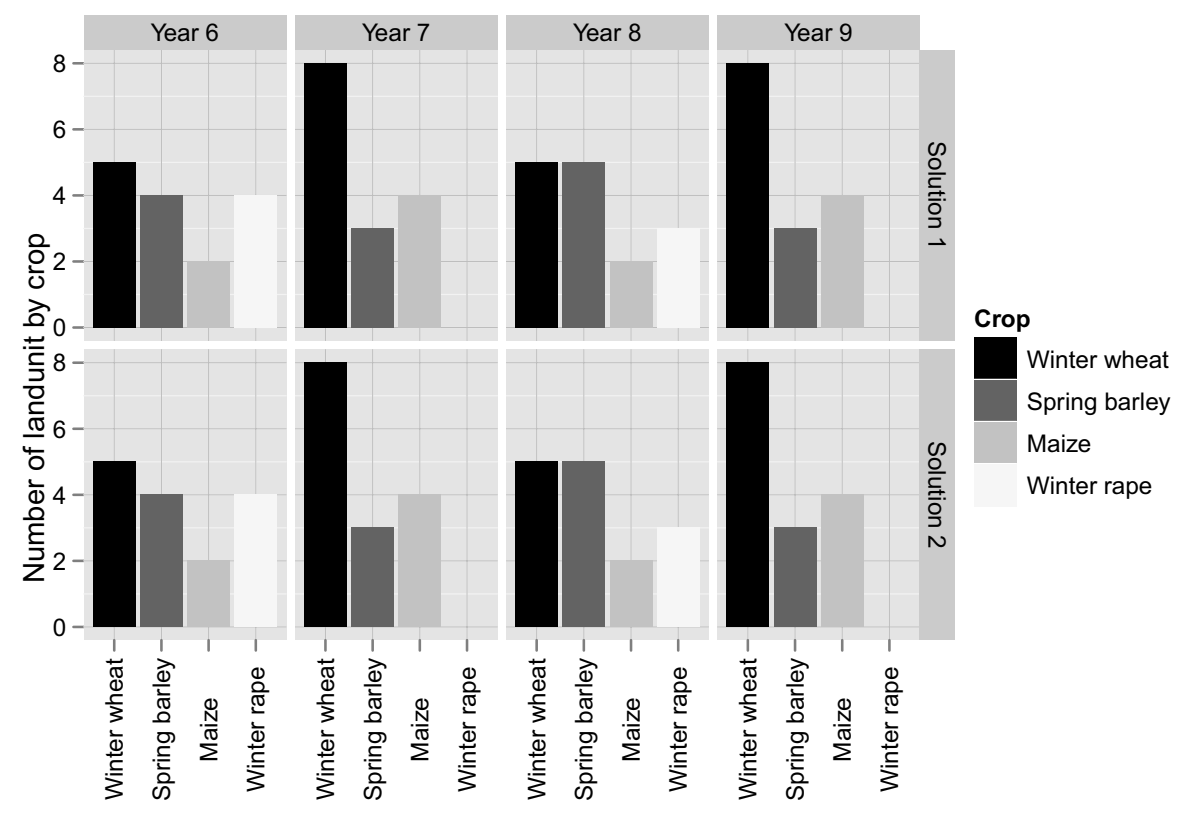

FiguRE 8. Spatial balance of crops over the years - B1234-LU15*.

optimum plus one (options $-\mathrm{f}=1-\mathrm{a}-\mathrm{ub}=0 \mathrm{ptimum}+1$, with variable elimination of 1 functional variables and other default options).

Let us consider the two optimal solutions found for the smallest instance B1234- 3 LU15*. Figure 8 shows that while considering a year and a crop, all the optimal 4 solutions have the same number of landunits assigned to the given crop. The spatial 5 balance of winter wheat is enforced contrary to the maize ones (year $t \in\{6,8\}$ ). 6 This is due to the historic values in block 3 .

We also represent in Figure 9, the spatio-temporal crop allocation over the 8 blocks. The graphic shows for each solution the crop succession over the farmland. 9 The minimum return times of crops are enforced. The difference between these 10 two solutions is related to the crop allocation in block 3. The spring barley (OP) 11 can be replaced by maize (MA) when the year $t \in\{7,9\}$. 


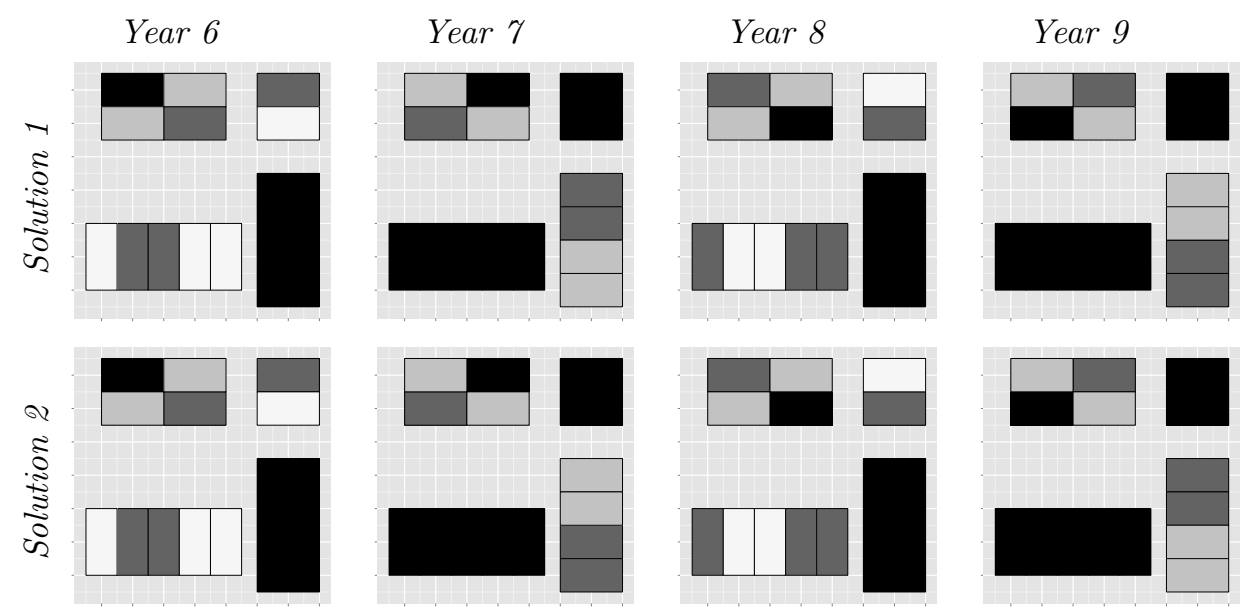

Figure 9. Crop allocation - B1234-LU15* : Winter wheat, Spring barley, Maize, Winter rape.

In this paper, we have modeled the crop allocation problem (CAP) using the Weighted CSP formalism. Contrary to existing approaches for solving such a problem, our proposition combines both the spatial and the temporal aspects of crop allocation. We explicitly described how the farmers' hard and soft constraints can be addressed as a mono-objective optimization problem. The results showed that on a medium-size CAP with 120 landunits over 180 ha and 9 years, despite the huge improvement obtained by a decomposed WCSP formulation compared to the direct one, the ILP formulation was more than three times faster than the best WCSP formulation and used less memory. ILP delivered relevant solutions in reasonable computation time, offering better scalability. However, the WCSP formalism offers a better abstraction of the crop allocation problem thanks to its global cost functions, also yielding improved flexibility for modeling possible problem changes. It suggests to develop a generic and automatic ILP reformulation of a WCSP as it is done in the NumberJack and MiniZinc [26] platforms for CSP.

By doing so, it will offer the modeling flexibility of WCSP and the solving efficiency of ILP.

In the future, we will investigate the soft CUMULATIVE constraint [31] for expressing more complex resource management situations. We will look at the combination of cost functions, e.g., the minimum return time REGULAR constraints with the preceding effects soft binary constraint in the spirit of [29]. Finally, we will perform experiments with the hybridization of WCSP and ILP solvers as introduced in [24]. 


\section{REFERENCES}

[1] D. Allouche, C. Bessiere, P. Boizumault, S. de Givry, P. Gutierrez, S. Loudni, JP. Métivier and T. Schiex, Decomposing Global Cost Functions, in Proc. AAAI-12, Toronto, Canada (2012).

[2] D. Allouche, S. Traoré, I. André, S. de Givry, G. Katsirelos, S. Barbe and T. Schiex, Computational protein design as a cost function network optimization problem, in Proc. CP-12, Quebec City, Canada (2012).

[3] J. Annetts and E. Audsley, Multiple objective linear programming for environmental farm planning. J. Operat. Res. Soc. 53 (2002) 933-943.

[4] K. Apt and S. Brand, Infinite Qualitative Simulations by Means of Constraint Programming, in Proc. CP-06, Nantes, France (2006) 29-43.

[5] J. Bachinger and P. Zander, ROTOR, a tool for generating and evaluating crop rotations for organic farming systems. Europ. J. Agron. 26 (2007) 130-143.

[6] N. Beldiceanu, I. Katriel and S. Thiel, Filtering algorithms for the same constraint, in Proc. CPAIOR-04, Nice, France (2004) 65-79.

[7] M.S. Castellazzi, J. Matthews, F. Angevin, C. Sausse, G.A. Wood, P.J. Burgess, I. Brown, K.F. Conrad and J.N. Perry, Simulation scenarios of spatio-temporal arrangement of crops at the landscape scale. Envir. Modell. Soft. 25 (2010) 1881-1889.

[8] M. Cooper, S. de Givry, M. Sanchez, T. Schiex, M. Zytnicki and T. Werner, Soft arc consistency revisited. Artificial Intell. 174 (2010) 449-478.

[9] S. Dogliotti, W.A.H. Rossing and M.K. van Ittersum, ROTAT, a tool for systematically generating crop rotations. Europ. J. Agron. 19 (2003) 239-250.

[10] J. Dury, The cropping-plan decision-making: A farm level modelling and simulation approach. PhD thesis, INP Toulouse, France (2011). http://ethesis.inp-toulouse.fr/ archive/00001788/01/dury.pdf

[11] J. Dury, N. Schaller,F. Garcia, A. Reynaud and JE. Bergez, Models to support cropping plan and crop rotation decisions. A review. Agron. Sustain. Develop. 32 567-580, 2012.

[12] T. El-Nazer and B.A. McCarl, The Choice of Crop Rotation: A Modeling Approach and Case Study. American J. Agricult. Econ. 68 (1986) 127-136.

[13] S. de Givry, M. Zytnicki, F. Heras and J. Larrosa, Existential arc consistency: Getting closer to full arc consistency in weighted CSPs, in Proc. IJCAI-05, Edinburgh, Scotland (2005).

[14] W.D. Harvey and M.L. Ginsberg, Limited discrepency search, in Proc. IJCAI-95, Montréal, Canada (1995).

[15] E.O. Heady, The Economics of Rotations with Farm and Production Policy Applications. J. Farm Econ. (1948) 645-664.

[16] W.J. van Hoeve, G. Pesant,L.M. Rousseau, On global warming: flow-based soft global constraints. J. Heurist. (2006) 347-373.

[17] S. Irnich and G. Desaulniers, Shortest Path Problems with Resource Constraints, chapter 2, GERAD 25th Anniversary Series. Springer (2005) 33-65.

[18] T. Itoh, H. Ishii and T. Nanseki, A model of crop planning under uncertainty in agricultural management. Int. J. Prod. Econ. 81-82 (2003) 555-558.

[19] W.K. Kein Haneveld and A.W. Stegeman, Crop succession requirements in agricultural production planning. Eur. J. Operat. Res. 166 (2005) 406-429.

[20] A. Koster, S. van Hoesel and A. Kolen. Solving frequency assignment problems via treedecomposition. Tech. Rep. RM/99/011, Universiteit Maastricht, The Netherlands (1999).

[21] J. Lee and K.L. Leung, Towards efficient consistency enforcement for global constraints in weighted constraint satisfaction, in Proc. IJCAI'09, Pasadena, CA (2009) 559-565.

[22] J. Lee and K.L. Leung, A stronger consistency for soft global constraints in weighted constraint satisfaction. in Proc. AAAI'10, Atlanta, GA (2010).

[23] J. Lee and K.M. Leung, Consistency techniques for flow-based projection-safe global cost functions in weighted constraint satisfaction. JAIR 43 (2012) 257-292. 
[24] J. Lee and Y.W. Shum, Modeling Soft Global Constraints as Linear Programs in Weighted Constraint Satisfaction, in Proc. ICTAI-11, Boca Raton, FL (2011) 305-312.

[25] B. Leteinturier, J. Herman, F. D. Longueville, L. Quintin and R. Oger, Adaptation of a crop sequence indicator based on a land parcel management system. Agriculture, Ecosystems and Environment 112 (2006) 324-334.

[26] K. Marriott, N. Nethercote, R. Rafeh, P. Stuckey, M. De La Banda and M. Wallace, The design of the Zinc modelling language. Constraints 13 (2008) 229-267.

[27] B. A. McCarl, W.V. Candler, D.H. Doster and P.R. Robbins, Experiences with farmer oriented linear programming for crop planning. Canadian J. Agricult. Econ./Revue canadienne d'agroeconomie 25 (1977) 17-30.

[28] P. Meseguer, F. Rossi and T. Schiex, Soft Constraints Processing, on edited by F. Rossi, P. van Beek and T. Walsh. Handbook Constraint Programm. chapter 9, Elsevier (2006).

[29] J.-P. Métivier, P. Boizumault and S. Loudni, Solving nurse rostering problems using soft global constraints, in Proc. CP-09, Lisbon, Portugal (2009) 73-87.

[30] G. Pesant, A regular language membership constraint for finite sequences of variables, in Proc. CP-04, Toronto, Canada (2004) 482-495.

[31] T. Petit and E. Poder, The Soft Cumulative Constraint. CoRR (2009).

[32] J.-C. Régin, Generalized arc consistency for global cardinality constraint, in Proc. AAAI'96, Portland, OR (1996) 209-215.

[33] M. Sánchez, S. de Givry and T. Schiex, Mendelian error detection in complex pedigrees using weighted constraint satisfaction techniques. Constraints 13 (2008) 130-154.

[34] R. Sarker and T. Ray, An improved evolutionary algorithm for solving multi-objective crop planning models. Comput. Electr. Agricul. 68 (2009) 191-199.

[35] N. Stone, R. Buick, J. Roach, R. Scheckler and R. Rupani, The planning problem in agriculture: farm-level crop rotation planning as an example. AI Appl. 6 (1992) 59-75. 